\title{
COMMUTATION WITH SKEW ELEMENTS IN RINGS WITH INVOLUTION
}

\author{
ChaRLes LANSKI
}

\begin{abstract}
This paper describes the structure of additive subgroups and of subrings which are invariant under Lie commutation with higher commutators of the skew-symmetric elements in 2-torsion free rings with involution. Except for cases arising when the subring is central, or when the ring satisfies a polynomial identity of small degree, the invariant subring must contain an ideal of the ring. With the same exceptions, the invariant subgroup must contain either the derived Lie ring of the set of skew-symmetric elements in some ideal, or the lie product of the set of skew-symmetric elements in the ideal with the set of symmetric elements in the ideal. Furthermore, the appropriate one of these Lie products is not Lie solvable.
\end{abstract}

The first general results of this kind were obtained for simple rings by Herstein [4], who characterized the Lie ideals of $K$, the set of skew-symmetric elements, and then by Baxter [2], who did the same for the Lie ideals of $[K, K]$, the derived ring of $K$. Their work has been extended in several ways. For prime rings, the Lie ideals of both $K$ and $[K, K]$ were studied by Erickson [3], and an investigation of additive subgroups of $K$ invariant under commutation with $[K, K]$ in semi-prime rings was made in [7]. This was followed by a description of arbitrary additive subgroups invariant under commutation with [K, K] [9], and of subgroups of $K$ invariant under commutation with higher commutators of $K[10]$. Returning to simple rings, Herstein [5] showed that no noncentral proper subring could be invariant under commutation with $K$, except in certain small dimensional cases. This work was extended to semi-prime rings and commutation with $[K, K]$ in [8]. Our purpose here is to complete this chain of results by describing the structure of additive subgroups and of subrings invariant under commutation with higher commutators of $K$.

Throughout the paper, $R$ will denote a 2 -torsion free ring with involution, *; $S(R)=S=\left\{r \in R \mid r^{*}=r\right\}$, the symmetric elements of $R ; K(R)=K=\left\{r \in R \mid r^{*}=-r\right\}$, the skew-symmetric elements of $R$; and $Z(R)=Z$, the center of $R$. The Lie product $[A, B]$ of subsets $A$ and $B$ of $R$ is the additive subgroup generated by all commutators $[a, b]=a b-b a$ for $a \in A$ and $b \in B$. A higher commutator of $K$ is a Lie product of $K$ with itself, some fixed number of times in a given association. For example, $[[K, K], K]=V$ is a higher commu- 
tator of $K$, as is $K^{(1)}=[K, K]$ or $\left[V, K^{(1)}\right]$. In general, write $K^{(i+1)}=$ $\left[K^{(i)}, K^{(i)}\right]$.

The goal of the theorems mentioned above is to show that Lie invariant additive subgroups of $K$ contain $[K(J), K]$ for $J$ a nonzero *-ideal of $R$, and that invariant subrings contain a nonzero *-ideal. Even for simple rings, one encounters two exceptions; when the invariant object is central, and when $R$ is no more than sixteen dimensional over its center. These exceptions exist for $R$ a prime ring also, and the second must include the possibility that $R$ is an order in such a simple ring, in which case we say that $R$ satisfies $S_{8}$. As one would expect for semi-prime rings, one of the three possibilities should hold in each prime image. In fact, a stronger result can be proved. In [10] it is shown that an invariant subgroup of $K$ contains $[K(J), K]$, which is "very" noncommutative or $R$ decomposes as a direct product of the two kinds of exceptions. To make these notions precise, we recall two definitions from [10].

Definition. Let $R$ be a 2-torsion free semi-prime ring and set $X=\left\{P \mid P\right.$ is a ${ }^{*}$-prime ideal of $R$ with $\left.2 R \not \subset P\right\}$. Let

$$
Q_{1}=\cap\left\{P \in X \mid R / P \text { does not satisfy } S_{8}\right\}
$$

and $Q_{2}=\cap\left\{P \in X \mid R / P\right.$ satisfies $\left.S_{8}\right\}$. If for some subset $T \subset R, T+$ $Q_{1} \subset Z\left(R / Q_{1}\right)$, then $\left(Q_{1}, Q_{2}\right)$ is called a splitting of $R$ for $T$.

When $A$ is an additive subgroup of $K$ invariant under commutation with some higher commutator of $K$, then to say that there is a splitting of $R$ for $A$ is clearly the same as being able to "construct" $R$ from the two kinds of exceptions discussed above. If no such splitting exists, one associates to $A a^{*}$-ideal of $R$ with the property described in our next definition.

Definition. Let $R$ be a 2-torsion free semi-prime ring, $A$ a subset of $R$, and $J$ a ${ }^{*}$-ideal of $R$. Then $J$ is called a controlling ideal for $A$ if for each $P \in X$ satisfying $K^{(i)}(J) \subset P$, either $R / P$ satisfies $S_{8}$ or $A+P \subset Z(R / P)$.

The existence of a controlling ideal for $A$ gives information about $A$ with respect to every $P \in X$. For example, if there were no splitting of $R$ for $A$, but $A \supset K^{(1)}(T)$ for some *-ideal $T$ of $R$ with $K^{(i)}(T) \neq 0$, one might have $T \subset P$ for some $P \in X$ with neither $A+P \subset Z(R / P)$ nor $R / P$ satisfying $S_{8}$. Even if $K^{(1)}(I+P) \subset A+P$ for a *-ideal $I+P$ of $R / P$, there is no obvious way to lift this ideal back to some $I$ in $R$ with $K^{(1)}(I) \subset A$, or to do this simultaneously for many primes. However, an ideal $J$ controlling $A$ with $A \supset K^{(1)}(J)$, 
uniformly satisfies $A+P \supset K^{(1)}(J+P)$ for every $P \in X$ and $K^{(i)}(J+P) \neq$ 0 unless $R / P$ is one of the two exceptional cases.

Next we make two easy observations to which we shall refer several times. Henceforth, we assume that for $P \in X$, the involution on $R / P$ is given by $(r+P)^{*}=r^{*}+P$.

LEMMA 1. Let $R$ be a semi-prime ring and $A$ an additive subgroup of $R$ satisfying $\left[A, K^{(i)}\right] \subset A$. Then for each $P \in X, K^{(1)}(R / P) \subset$ $K(R)+P$, and so, $\left[A+P, K^{(i+1)}(R / P)\right] \subset A+P$.

Proof. Clearly, it suffices to show that $K^{(1)}(R / P) \subset K(R)+P$. But if $x+P, y+P \in K(R / P)$, then $(x y-y x)+P=\left(x y-y^{*} x^{*}\right)+P \in$ $K(R)+P$.

LEMMA 2. Let $R$ be a semi-prime ring and $J a{ }^{*}$-ideal of $R$. If for some $P \in X, K^{(i)}(J) \subset P$, then either $J \subset P$ or $R / P$ satisfies $S_{8}$.

Proof. If $J \not \subset P, J+P$ is a nonzero *-ideal of $R / P$ with $K^{(i)}(J+P)=0$. It can be shown that this condition forces $J+P$ to satisfy $S_{4}$, although one can get directly that $J+P$ satisfies $S_{8}$ by using Lemma 1, applying [10; Lemma 3], and then applying [7; Lemma 2, p. 735]. It follows that $R / P$ must satisfy $S_{8}$ since it has an ideal which does.

Before our first main result, which extends [9; Theorem 1, p. 77] to higher commutators, note that if $V$ is any higher commutator of $K$, then $V \subset K$ and $[V, K] \subset V$. An essential ingredient in our arguments is [10; Theorem 1] applied to higher commutators of $K$, which we state as

THEOREM A. Let $R$ be a semi-prime ring and $V$ a higher commutator of $K$. There exists an ideal $I^{*}=I$ of $R$ which is a controlling ideal for $V$, and which satisfies $V \supset[K(I), K]$ and $\bar{V} \supset I$, where $\bar{V}$ is the subring generated by $V$.

With the preliminaries done, we can now prove our first main result, about invariant additive subgroups of $S$.

THEOREM 1. Let $R$ be a semi-prime ring, $A$ an additive subgroup of $S$, and $V$ a higher commutator of $K$ so that $[A, V] \subset A$. Then either there is a splitting of $R$ for $A$, or there exists $a^{*}$-ideal $I$ of $R$ controlling $A$ with $A \supset[K(I), S(I)]=Y$ and $Y^{(i)} \neq 0$ for any $i$.

Proof. By Theorem A, $V \supset[K(J), K]$ for $J^{*}=J$, an ideal of $R$ controlling $V$. Let $B=J \cap A$, and observe that $\left[B, K^{(1)}(J)\right] \subset B$, and 
that $J$ is a semi-prime ring. Using [9; Theorem 1, p. 77] we may conclude that either there is a splitting of $J$ for $B$, or that there exists an ideal $T^{*}=T$ of $J$ with $B \supset[S(T), K(T)]$. The last paragraph of the proof of [9; Theorem 1, p. 81] shows that for any $P \in X(J)$ with $T \subset P$, either $J / P$ satisfies $S_{8}$ or $B+P \subset Z(J / P)$. This together with Lemma 2, shows that $T$ is a controlling ideal for $B$.

Assume first that there is a splitting of $J$ for $B$. It follows that there is a splitting of $R$ for $B$ [10; Theorem 2]. Hence, for each $P \in X$ either $R / P$ satisfies $S_{8}$ or $(A \cap J)+P \subset Z(R / P)$. Since $\left[A, K^{(1)}(J)\right] \subset A \cap J$, one obtains $\left[A, K^{(2)}(J)\right] \subset P$, if $R / P$ does not satisfy $S_{8}$. Should $K^{(i)}(J) \subset P$, then because $J$ is a controlling ideal for $V, V+P \subset Z(R / P)$. An easy induction argument shows that $K^{(j)} \subset V$ for some $j$, so that $K^{(j+1)} \subset P$. The fact that $P$ is a proper ideal of $R$ and Lemma 2 give that $R / P$ must satisfy $S_{8}$. On the other hand, if $K^{(i)}(J) \not \subset P$, then $K^{(2)}(J)+P$ is not commutative and $\left[K^{2}(J)+P\right.$, $\left.K^{(1)}(R / P)\right] \subset K^{(2)}(J)+P$ by Lemma 1, so [8; Theorem 2, p. 90] may be used to conclude that the subring generated by $K^{(2)}(J)+P$ contains a nonzero *-ideal of $R / P$, unless $R / P$ satisfies $S_{8}$. Thus the condition $\left[A, K^{2}(J)\right] \subset P$ forces either $R / P$ to satisfy $S_{8}$ or $A+P \subset Z(R / P)$. Consequently, a splitting of $J$ for $B$ gives rise to a splitting of $R$ for $A$.

Next, assume that $A \supset B \supset[S(T), K(T)]$, for $T$ an ideal of $J$ controlling $B$. Set $I=J T J$, a *ideal of $R$. Clearly, $A \supset[S(I), K(I)]$ and we claim that $I$ is a controlling ideal for $A$. Let $P \in X$ and suppose that $K^{(i)}(I) \subset P$. By Lemma 2, either $R / P$ satisfies $S_{8}$ or $I \subset P$. Assuming that $R / P$ does not satisfy $S_{8}$, the *-primeness of $P$, together with the facts that $J$ is a ${ }^{*}$-ideal of $R$, and $T^{*}=T \subset J$, gives $T \subset P \cap J$. If $J \not \subset P$ then $P \cap J \in X(J)$, so $T \subset P \cap J$ means that $J / P \cap J$ satisfies $S_{8}$ or $B+(P \cap J) \subset Z(J / P \cap J)$, since $T$ is a controlling ideal for $B$. The first possibility is equivalent to the nonzero ideal $J+P$ of $R / P$ satisfying $S_{8}$, which would force $R / P$ to satisfy $S_{8}$. In the second case, $(A \cap J)+P \subset Z(R / P)$ and our argument in the last paragraph shows that $A+P \subset Z(R / P)$ if $R / P$ does not satisfy $S_{8}$. The same argument shows that $R / P$ must satisfy $S_{8}$ when $J \subset P$. By definition, $I$ is a controlling ideal for $A$.

Finally, assume that $Y^{(i)}=0$ for $Y=[S(I), K(I)]$. We claim that this gives a splitting of $R$ for $A$. Let $P \in X$ and note that $Y^{(i)}+P \subset P$, $Y^{(1)}+P \subset K(R / P)$, and by Lemma $2\left[Y^{(1)}+P, K^{(1)}(R / P)\right] \subset Y^{(1)}+P$. From [10; Lemma 3] we have either $Y^{(1)}+P \subset Z(R / P)$ or that $R / P$ satisfies $S_{8}$. In the first case, a result of Amitsur [1; Theorem 1, p. 63] shows that $(I+P) / P$ satisfies a polynomial identity, and so, $R / P$ satisfies the same identity. Of course, if $I \subset P$ we would be finished by our earlier arguments. Consequently, localizing $R / P$ at its central symmetric elements gives a semi-simple finite dimensional algebra $Q$ [6]. 
Since in this localization, $I+P$ becomes $Q, S(I)+P$ localizes to $S(Q)$, and $K(I)+P$ localizes to $K(Q)$, it follows that in $Q,[[S, K],[S, K]] \subset$ $Z(Q)$. A consideration of the possible cases shows that $Q$ is at most four dimensional over its center. Very briefly, if $Q$ is not simple, or has an involution of the second kind, then $Q^{(3)}=0$, and otherwise one can split $Q$ to obtain matrices over a field, where straightforward computations give the result. Consequently, $R / P$ must satisfy $S_{8}$ (in fact, $S_{4}$ ) so $Y^{(i)}=0$ forces a splitting of $R$ for $A$, completing the proof of the theorem.

Combining Theorem 1 with [10; Theorem 4] gives the version of [9; Theorem 2, p. 82] for higher commutators of $K$.

THEOREM 2. Let $R$ be a semi-prime ring, $V$ a higher commutator of $K$, and $A$ an additive subgroup of $R$ satisfying $[A, V] \subset A$. Then one of the following holds:

(i) $A \supset[K(I), K]=L$ for $I^{*}=I$ an ideal of $R$ controlling $A \cap K$, and $L^{(i)} \neq 0$;

(ii) $A \supset[K(I), S(I)]=Y$ for $I^{*}=I$ an ideal of $R$ controlling $A \cap S$, and $Y^{(i)} \neq 0$;

(iii) there is a splitting of $R$ for $A \cap S+A \cap K$.

If in addition, $A^{*}=A$, then (iii) can be replaced by: (iii)' there is a splitting of $R$ for $A$.

In trying to improve Theorem 2 (iii) to (iii)', the same counterexample and considerations as in [9] show that some additional assumption is required. Before discussing the nature of the involution on $R$, we point out that if in Theorem 2 (iii), for each $P \in X$ with $R / P$ not satisfying $S_{8}, P$ is not a prime ideal of $R$, then in fact $A+P \subset$ $Z(R / P)$. To prove this, note first that if $P$ is not a prime ideal of $R$, then $P=Q \cap Q^{*}$ for a $Q$ prime ideal of $R$. Now $Q+Q^{*}$ is a nonzero ideal of $R / Q^{*}$ and $q+Q^{*}=\left(q-q^{*}\right)+Q^{*}$, so $Q+Q^{*} \subset K+Q^{*}$. If the higher commutator $V$ in Theorem 2 contains $K^{(i)}$, then $\left[A, Q^{(i)}\right]+$ $Q^{*} \subset\left[A, K^{(i)}\right]+Q^{*} \subset A+Q^{*}$, so $\left[A, Q^{(i)}\right]+Q^{*} \subset(A \cap Q)+Q^{*} \subset$ $(A \cap K)+Q^{*} \subset Z\left(R / Q^{*}\right)$. Since $Q^{(i)}+Q^{*}$ is a lie ideal in $R / Q^{*}$, it follows that either $A+Q^{*} \subset Z\left(R / Q^{*}\right)$, or $Q^{(i)}+Q^{*} \subset Z\left(R / Q^{*}\right)$, unless $R / Q^{*}$ satisfies $S_{4}$ [11; Lemma 8, p. 120]. The possibility $Q^{(i)}+Q^{*} \subset Z\left(R / Q^{*}\right)$ and repeated use of [11; Lemma 7, p. 120] force $Q+Q^{*} \subset Z\left(R / Q^{*}\right)$, which in turn means that $R / Q^{*}$ is commutative. Repeating the whole argument with $Q$ and $Q^{*}$ interchanged shows that $A+P \subset$ $Z(R / P)$ unless $R / P$ satisfies $S_{4}$. We isolate one special case of Theorem 2 to which our observation applies.

COROLlary. If in Theorem 2, $R$ is $a^{*}$-prime ring which is not prime, then $A \cap S+A \cap K \subset Z$ forces $A \subset Z$ unless $R$ satisfies $S_{8}$. 
As in [9], the obstruction to showing that a splitting of $R$ for $A \cap S+A \cap K$ forces a splitting of $R$ for $A$ occurs in prime rings whose extended centroid has an induced involution of the second kind [13; Theorem 4.1, p. 511]. When this involution is of the first kind, we can prove the result corresponding to [9; Theorem 7, p. 93] for higher commutators.

THEOREM 3. Let $R$ be a prime ring with extended centroid $C$, and assume that the involution induced on $C$ is the identity map. If $V$ is a higher commutator of $K$ and $A$ is an additive subgroup of $R$ satisfying $[A, V] \subset A$, then $A \cap S+A \cap K \subset Z$ implies that either $A \subset Z$ or $R$ satisfies $S_{8}$.

Proof. Let $I$ be the controlling ideal for $V$ given by Theorem A. Then $I$ is a prime ring and $K^{(1)}(I) \subset V$ implies that $\left[A \cap I, K^{(1)}(I)\right] \subset$ $A \cap I$. We wish to apply [9; Theorem 7, p. 93] to $I$ and $A \cap I$, but first we must verify that the involution on $C_{I}$, the extended centroid of $I$, is the identity map. This follows from work of Martindale since the extended centroid is the center of a certain quotient ring and these quotient rings coincide for $R$ and for $I$ [12; Theorem 1 , p. 440]. A proof of this result, using the definitions in [13] follows easily from the fact that any ideal $T$ of $I$ contains the ideal ITI of $R$ and an ideal $N$ of $R$ contains the ideal $N I$ of $I$. This observation and [13; proof of Theorem 4.1, p. 511-512] show that $C$ and $C_{I}$ have the same kind of involution. Applying [9; Theorem 7, p. 93] gives either $A \cap I \subset Z(I)$ or $I$ satisfies $S_{8}$. Since $I$ satisfying $S_{8}$ forces $R$ to satisfy $S_{8}$, assume that $A \cap I \subset Z(I)$. Thus $A \cap I \subset Z(R)$, and in particular $\left[A, K^{(1)}(I)\right] \subset Z$, forcing $\left[A, K^{(2)}(I)\right]=0$. As in the first part of the proof of Theorem 1, we must have $A \subset Z$ unless $R$ satisfies $S_{8}$, completing the proof of the theorem.

Using the same ideas as above, we can obtain the higher commutator version of [8; Theorem 3, p. 92] for invariant subrings. Note that for subrings, the nature of the involution is immaterial.

THEOREM 4. Let $R$ be a semi-prime ring, $V$ a higher commutator of $K$, and $A$ a subring of $R$ satisfying $[A, V] \subset A$. Then either $A \supset M^{*}=M$, a noncommutative ideal of $R$ controlling $A$, or there is a splitting of $R$ for $A$.

Proof. By Theorem A, $V \supset[K(I), K]$ for $I^{*}=I$ an ideal of $R$ controlling $V$. Clearly, $B=A \cap I$ satisfies $\left[B, K^{(1)}(I)\right] \subset B$, so [8; Theorem 3, p. 92] applies to the subring $B$ of $I$ to yield a splitting of $I$ for $B$, or that $B \supset T^{*}=T$, a noncommutative ideal of $I$. We 
observe that the proof of [8; Theorem 3, p. 92] actually shows that $T$ is a controlling ideal for $B$, since $T$ can be chosen to be a controlling ideal for $B \cap K$ by [10; Theorem 1], which is all that is necessary. If $B \supset T$ holds, then $A \supset B \supset I T I=M$. The fact that $M$ is a controlling ideal for $A$ follows exactly as in the proof of Theorem 1. Should $M$ be commutative, the semi-primeness of $R$ would force $M \subset Z(R)$. In particular, $T^{3} \subset Z(T)$ and $Z(T)^{2} T \subset Z(T)$. Thus $0=\left[Z(T)^{2} T, T\right]=Z(T)^{2}[T, T]$, so $Z(T)[1, T]=0$ from the fact that $T$ is a semi-prime ring. But now $T^{3}[T, T]=0$ so that $(T[T, T])^{2}=$ 0 , forcing $T[T, T]=0$. Hence $[T, T] \subset T \cap \operatorname{Ann}(T)=0$, contradicting the assumption that $T$ is not commutative. To complete the proof of the theorem, it suffices to treat the case when there is splitting of $I$ for $B$. As in the proof of Theorem 1, such a splitting gives a splitting of $R$ for $B$ [10; Theorem 2], and the fact that $B \supset$ $\left[A, K^{(1)}(I)\right]$ for $I$ a controlling ideal of $R$ for $V$ yields a splitting of $R$ for $A$.

\section{REFERENCES}

1. S. A. Amitsur, Identities in rings with involutions, Israel J. Math., 7 (1969), 63-68. 2. W. Baxter, Lie simplicity of a special class of associative rings II, Trans. Amer. Math. Soc., 87 (1958), 63-75.

3. T. Erickson, The Lie structure in prime rings with involution, J. of Algebra, 21 (1972), 523-534.

4. I. N. Herstein, Lie and Jordan systems in simple rings with involution, Amer. J. Math., 78 (1956), 629-649.

5. - Certain submodules of simple rings with involution, II, Canad. J. Math., 27 (1975), 629-635.

6. N. Jacobson, PI-Algebras, Lecture Notes in Mathematics No. 441, Springer-Verlag, New York, 1975.

7. C. Lanski, Lie structure in semi-prime rings with involution, Comm. in Algebra, 4 (1976), 731-746.

8. - Invariant subrings in rings with involution, Canad. J. Math., 30 (1978), $85-94$.

9. - Invariant submodules in semi-prime rings with involution, Comm. in Algebra, 6 (1978), 75-96.

10. - Lie structure in semi-prime rings with involution, II, Comm. in Algebra, 6 (1978), 1755-1775.

11. C. Lanski and S. Montgomery, Lie structure of prime rings of characteristic 2, Pacific J. Math., 42 (1972), 117-136.

12. W.S. Martindale, 3rd, Lie isomorphisms of prime rings, Trans. Amer. Math. Soc., 142 (1969), 437-455.

13. - Prime rings with involution and generalized polynomial identities, J. of Algebra, 22 (1972), 502-516.

Received December 4, 1978 and in revised form March 5, 1979. This work was supported by NSF Grant MCS 78-01491.

University of Southern California

LOS ANGELES, CA 90007 
